\title{
Radiographic Diagnosis of Scapholunate Diastasis in Distal Radius Fractures: Implications for Surgical Practice
}

\author{
Sezai Özkan, MD ${ }^{1,2}$ Julian J. Korteweg, BSc ${ }^{1}$ Frank W. Bloemers, MD, PhD ${ }^{2}$
} Nicholas C. DiGiovanni, BSc ${ }^{3}$ Chaitanya S. Mudgal, MD, MS, MCh ${ }^{1}$

${ }^{1}$ Hand and Upper Extremity Service, Department of Orthopedic Surgery, Massachusetts General Hospital, Harvard Medical School, Boston, Massachusetts

2 Department of Trauma Surgery, VU University Medical Center, VU University, Amsterdam, The Netherlands

${ }^{3}$ Harvard College, Harvard University, Cambridge, Massachusetts
Address for correspondence Chaitanya S. Mudgal, MD, MS, MCh, Hand and Upper Extremity Service, Department of Orthopedic Surgery, Massachusetts General Hospital, Harvard Medical School, Yawkey Center for Outpatient Care, Suite 2100, 55 Fruit Street, Boston, MA 02114 (e-mail: cmudgal@partners.org).

J Wrist Surg 2018;7:312-318.

\begin{abstract}
Background Radiographic diagnosis of scapholunate ligament injury (SLI) in the setting of distal radius fractures (DRFs) is challenging. It remains unclear to what extent radiographic diagnosis of SLI by a radiologist influences surgical decision-making regarding treatment of SLI.

Purpose We aimed to (1) identify the number of times that concerns for the possibility of concurrent SLI in the setting of a DRF had been raised by the radiologists, (2) identify how often the radiologist's diagnosis was confirmed by the treating surgeon, and (3) how many of the patients with a radiographic concern for SLI by the radiologist received operative treatment for the SLI.

Patients and Methods Based on Current Procedural Terminology codes, we identified 2,923 patients that were operatively treated for their DRF in 1 of 3 participating institutions in an urban city in the United States. We reviewed the medical charts of 654 patients who had a mention of scapholunate ligament (SL) distance in their radiography, surgery, or clinical notes. We then measured the SL distance and recorded patient, diagnosis, and treatment characteristics of all these patients.

Results A total of 200 out of 2,923 patients (6.8\%) received a radiological diagnosis of

Keywords

- distal radius fractures

- scapholunate diastasis

- radiographic diagnosis SLI. In seven of these patients (3.5\%), the surgeon confirmed the diagnosis of the radiologist. Four patients (2\%) had operative repair of their SLI.

Conclusion Radiologists demonstrate a low threshold to identify SLI in the setting of DRFs, while the number of SLIs identified by the treating surgeon is a remarkably smaller number.

Level of Evidence Level II, prognostic study.
\end{abstract}

Distal radius fractures (DRFs) are often accompanied by concomitant ligamentous injury in the wrist. ${ }^{1}$ The most commonly associated intercarpal ligament lesion is scapholunate ligament injury (SLI), with reported incidence rates varying from 5 to $69 \% .^{2-4}$ This rate seems to be higher in intra-articular DRFs or fractures with a positive ulnar variance that is more than $2 \mathrm{~mm} .{ }^{5}$ Despite our evolving understanding of the pathomechanics of these injuries, ${ }^{6,7}$ clinical received

December 24, 2017

accepted

April 17, 2018

published online

May 23, 2018
Copyright $\odot 2018$ by Thieme Medical

Publishers, Inc., 333 Seventh Avenue, New York, NY 10001, USA Tel: +1(212) 584-4662.
DOI https://doi.org/ $10.1055 / \mathrm{s}-0038-1654699$. ISSN 2163-3916. 
diagnosis of associated SLI is challenging due to localized pain, and diagnosis of acute concomitant SLI depends largely on radiographic assessment. ${ }^{8}$ It has been suggested that radiographic evaluation of SLI is better suited for excluding rather than diagnosing SLI. ${ }^{9}$ In addition, the outcomes of posttraumatic radiographic assessment of SLI should be interpreted with caution because patients with SLI may have a normal radiographic appearance or the SLI may have a nontraumatic origin. ${ }^{10,11}$ Nevertheless, patients with a DRF accompanied by SLI seem to benefit from timely diagnosis and treatment. ${ }^{8}$ There is, however, insufficient evidence regarding the choice of surgical treatment of SLI and treatment considerations made by surgeons are therefore mostly experience-based rather than evidence-based. ${ }^{12}$ Since closed treatment of DRFs appears to be losing popularity, we speculate that we might see a progression of SLI that would be treated implicitly through immobilization of the DRF. ${ }^{5}$ Given the fact that it remains unclear to what extent radiographic diagnosis of SLI associated with a DRF should influence the surgical decision-making regarding the treatment of these ligamentous injuries, we aimed to identify the number of times that concerns about the possibility of concurrent SLI in the setting of a DRF had been raised by the radiologists, and to identify the number of patients who had a clinically confirmed diagnosis of this radiographically established concern for SLI. We further aimed to identify how many of the patients with a radiographic concern by the radiologist for SLI had an operative scapholunate ligament (SLL) repair. Finally, we aimed to identify risk factors that are (independently) associated with operative repair of SLL diastasis in patients who were operatively treated for a DRF.

\section{Patients and Methods}

\section{Study Design}

After approval by our institutional review board, we queried a research database of three affiliated urban hospitals in a single city in the United States from 2005 to 2015. We retrospectively included all patients who were at least 18 years old at the time of surgical treatment of their DRF, as identified by the Current Procedural Terminology (CPT) codes for the treatment of DRFs by means of external fixation (20690 or 20692), percutaneous Kirschner wire pinning (25606), or open reduction internal fixation (ORIF) (25607-25609). This initial query resulted in 2,923 patients. We assessed their radiography, surgery, and clinical reports for the presence of (variations or fragments of) the word "scapholunate" and "SL," resulting in 654 patients. To make sure we would not miss patients who were treated for their scapholunate ligament (SL) diastasis but might have had miscoding of their DRF, we cross-matched this cohort by performing the same query based on CPT codes for operative or nonoperative treatment of SL diastasis $(25320,25660$, and 25670), resulting in an additional 47 patients. We then manually reviewed the radiography, surgery, and clinical notes of these patients from their medical charts and established an initial cohort of 291 patients who had a diagnosis of
SL diastasis by the radiologist on any radiograph taken in the course of treatment of the DRF. Based on manual chart review, we recorded characteristics related to the patient (age, sex, obesity, smoking status, alcohol abuse), the injury (trauma mechanism, injured hand, open or closed fracture), and the diagnosis and treatment (type of treatment, time from injury to treatment, time from injury to radiographic diagnosis of SLI). One author (S.Ö.) measured the SL gap on the first radiograph that was reported to be positive for SL diastasis, which was measured from the midpoints of the opposing facets of the scaphoid and the lunate on the posteroanterior radiograph of the wrist. These measurements were for informative purposes only-inclusion in the cohort was based on the radiologist's diagnosis, and not on our own measurements.

After excluding patients based on our exclusion criteria (-Table 1), our final cohort consisted of 200 patients who had surgical treatment of their DRF and had a diagnosis of SL diastasis by the radiologist based on the radiographs of the wrist (-Fig. 1). The median follow-up duration was 24 weeks (interquartile range [IQR]: 15-42 weeks). The mean follow-up of the patients who had treatment for their SLI was $21 \pm 9.7$ weeks, whereas this was $39 \pm 49$ weeks for the patients who did not have treatment for their SLI.

\section{Patient Characteristics}

Our study sample consisted of 148 women (74\%) and 52 men (26\%) and the mean age was $58 \pm 14$ years. The mechanism of injury had a low-energy origin (fall from standing height

Table 1 Exclusions

\begin{tabular}{|l|l|}
\hline Exclusion reason & $n$ \\
\hline $\begin{array}{l}\text { Diagnosis on CT/MRI prior to } \\
\text { radiographic diagnosis }\end{array}$ & 22 \\
\hline Initial treatment at other institution & 13 \\
\hline Incomplete chart information & 2 \\
\hline Images not retrievable from PACS & 11 \\
\hline Lost to follow-up & 1 \\
\hline $\begin{array}{l}\text { Prior distal radius fracture of same } \\
\text { extremity (refracture) }\end{array}$ & 4 \\
\hline Carpal fracture & 15 \\
\hline Nonoperative treatment & 3 \\
\hline $\begin{array}{l}\text { No distal radius fracture (radiographically } \\
\text { occult fracture) }\end{array}$ & 4 \\
\hline Perilunate dislocation & 2 \\
\hline $\begin{array}{l}\text { No clear AP radiograph for measuring } \\
\text { SL distance }\end{array}$ & 13 \\
\hline $\begin{array}{l}\text { SL widening prior to distal radius fracture } \\
\text { (on old radiograph) }\end{array}$ & 1 \\
\hline Total number of exclusions & 91 \\
\hline
\end{tabular}

Abbreviations: AP, anteroposterior; $\mathrm{CT}$, computed tomography; MRI, magnetic resonance imaging; PACS, picture archiving and communication system; SL, scapholunate ligament. 


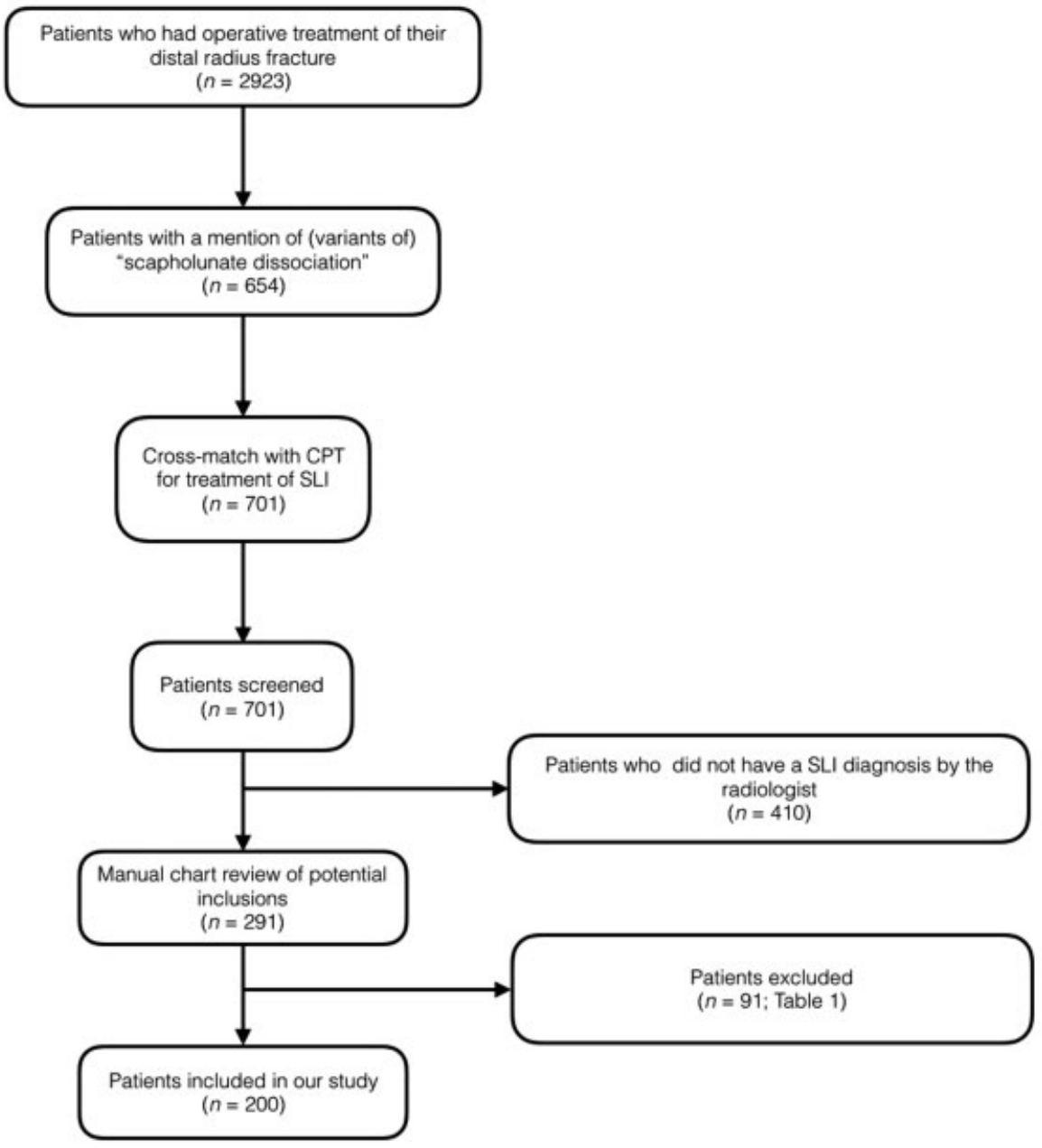

Fig. 1 Flowchart of patient inclusions.

or less) in a majority of patients $(n=159 ; 80 \%)$ and a highenergy origin (fall from height higher than standing height, motor vehicle accidents, or sports injuries) in 40 patients (20\%). One patient $(0.50 \%)$ had a fracture that was caused by a crush injury. Ninety percent of the patients had ORIF of their DRF. The first time SLI was diagnosed was on the postoperative follow-up radiographs in the majority of the patients: $36 \%$ (72 patients) of the SLI diagnosis were made on the first postoperative radiograph, and $40 \%$ (79 patients) on a later postoperative follow-up radiograph. Of the 49 out of 200 patients (25\%) who received a diagnosis of SLI preoperatively, 37 patients (19\%) were diagnosed on the postreduction radiograph and 12 patients (6\%) were diagnosed on the prereduction radiograph.

\section{Statistical Analysis}

We described the frequencies of dichotomous and categorical variables. Continuous variables with a normal distribution were reported as mean values and standard deviations (SDs). We reported the median and IQR for continuous variables with a nonparametric distribution. We initially planned to perform a bivariate and a multivariable analysis to identify factors that are (independently) associated with operative treatment of SL diastasis, but, unfortunately, this was not feasible due to the low number of patients who had operative treatment of their SL diastasis.

\section{Results}

Of the 2,923 patients that had operative treatment of their DRF, 654 patients (22\%) had a mention of variations or fragments of the term "scapholunate" or "SL" in the reports of their plain radiographs, including (variations of) "normal scapholunate distance." Of these 654 patients, 200 patients (30\%) had a diagnosis of SLI on their radiographic report, dictated by the radiologist. Seven of these patients (3.5\%) had a clinical concern for SLI in their visit note, as written by the treating surgeon. Except for 1 patient whose first mention of SLI was in the operative report, all descriptions regarding SLI in these 7 patients were based on the radiograph report that was dictated by the radiologist. The mean SL distance on the first radiograph indicating SLI in these 7 patients was $4.2 \pm 1.5 \mathrm{~mm}$ (range, $2.3-6.1 \mathrm{~mm}$ ). Of the 200 patients who had operative treatment of their DRF and a diagnosis of SLI, 196 patients (98\%; - Table 2 ) had no repair of their SLI and 4 patients $(2 \%$; - Table 3 ) did. Of the three patients who 
Table 2 Characteristics of patients with radiographical SL diastasis, but no SL reconstruction ( $n=196$ patients)

\begin{tabular}{|c|c|}
\hline Variable & \\
\hline \multicolumn{2}{|l|}{ Patient characteristics } \\
\hline Age, mean $\pm S D$, years & $58 \pm 14$ \\
\hline \multicolumn{2}{|l|}{ Sex, $n(\%)$} \\
\hline Men & $50(26)$ \\
\hline Women & $146(74)$ \\
\hline Obesity, $n(\%)$ & $65(33)$ \\
\hline Alcohol abuse, $n(\%)$ & $18(9.2)$ \\
\hline Smoking, $n(\%)$ & $20(10)$ \\
\hline \multicolumn{2}{|l|}{ Trauma characteristics } \\
\hline High energy wrist trauma, $n(\%)$ & $38(19)$ \\
\hline Dominant hand injured, $n(\%)$ & $81(41)$ \\
\hline Open fracture, $n(\%)$ & $10(5.1)$ \\
\hline \multicolumn{2}{|l|}{ Diagnosis and treatment characteristics } \\
\hline $\begin{array}{l}\text { Days from injury to distal radius surgery, } \\
\text { mean } \pm S D\end{array}$ & $9.2 \pm 9.9$ \\
\hline $\begin{array}{l}\text { Days from injury to first } S L+\text { radiograph, } \\
\text { median (IQR) }\end{array}$ & $30(8.5-80)$ \\
\hline \multicolumn{2}{|l|}{ Type of DRF $\times$ treatment, $n(\%)$} \\
\hline ORIF & $176(90)$ \\
\hline ORIF + pinning & $5(2.6)$ \\
\hline ORIF + screws & $4(2.0)$ \\
\hline Kirschner wire & $4(2.0)$ \\
\hline External fixator & $4(2.0)$ \\
\hline Screw fixation & $3(1.5)$ \\
\hline \multicolumn{2}{|l|}{ Radiographic characteristics } \\
\hline \multicolumn{2}{|l|}{$\begin{array}{l}\text { Type of radiograph for first diagnosis, } \\
n(\%)\end{array}$} \\
\hline Pre-red & $11(5.6)$ \\
\hline Post-red & $35(18)$ \\
\hline Post-op & $72(37)$ \\
\hline Post-op follow-up & $78(40)$ \\
\hline \multicolumn{2}{|l|}{$\begin{array}{l}\text { Measured SL diastasis on first SL+ } \\
\text { radiograph, mean } \pm \text { SD, mm (\%) }\end{array}$} \\
\hline Pre-red & $2.8 \pm 0.62$ \\
\hline Post-red & $3.7 \pm 1.4$ \\
\hline Post-op & $4.1 \pm 1.5$ \\
\hline Post-op follow-up & $3.7 \pm 1.2$ \\
\hline
\end{tabular}

Abbreviations: DRF, distal radius fracture; IQR, interquartile range; ORIF, open reduction internal fixation; SD, standard deviation; SL, scapholunate ligament.

had a clinical concern of SL diastasis but no operative treatment for their SLI, the surgeon did not feel the treatment was warranted based on the intraoperative fluoroscopy. None of the 196 patients without operative treatment of their SLI have undergone a SL repair so far. Due to the low number of patients who had a SL repair, a statistical analysis was not feasible and we were therefore unable to identify factors that were (independently) associated with SL repair.

\section{Discussion}

Radiographic diagnosis of SLI in the setting of DRFs can be challenging, even when combined with adequate history taking and proficient clinical assessment of tenderness over the dorsal aspect of the scapholunate articulation. Our aims were to understand and identify the incidence of radiographic diagnosis of concurrent SL diastasis in the setting of a DRF. We further aimed to identify how often the radiologist's diagnosis was confirmed by the treating surgeon and how many of the patients with a concern for SLI by the radiologist received operative treatment for the SLI. Finally, we aimed to identify the risk factors that are (independently) associated with operative repair of SL diastasis in patients who were operatively treated for a DRF.

This study should be interpreted in light of its strengths and limitations. First, our study comprises patients from two level I trauma centers and one affiliated community hospital in an urban city in the United States and our findings may not be applicable to other settings. Second, since this is a retrospective study, we relied on accurate recordkeeping and there may be an element of misregistration. This, however, is typical for retrospective chart studies and is unlikely to influence our outcomes. ${ }^{13}$ Third, even though large database studies such as this study are an appropriate study design to investigate relatively uncommon events such as SL repair surgery in DRFs, this type of surgery was too uncommon to facilitate a statistically meaningful analysis of factors that may have an (independent) association with SL repair surgery. Finally, while definitive criteria for the diagnosis of SLI by the radiologists in our study were not reported in the radiology reports, we can only speculate based on personal communications that the cut-off value of $2 \mathrm{~mm}$ was utilized by the radiologists. The main strengths of our study comprise our large study sample and our manual chart review, providing us chart-abstracted data which allows evaluation for clinical details rather than information based on databasecoding alone.

Numerous studies have reported varying incidences of SLI associated with DRFs, based on either medical imaging or arthroscopy of the wrist. ${ }^{1-5,14-16}$ The incidence of specifically radiographically diagnosed SLI in patients who were operatively treated for DRFs seems to lie between 8.1 and $32 \%{ }^{2,15}$ Although radiographs are considered to be a reliable tool in diagnosing $\operatorname{SLI},{ }^{17,18}$ plain radiographs seem to be more useful for excluding rather than confirming SLI. ${ }^{9}$

In this study, 200 out of the 2,923 patients (6.8\%) who were operatively treated for their DRF had signs of SLI on their plain radiographs taken in the course treatment of the DRF (- Table 2), as diagnosed by a radiologist. Surgeons mentioned a concern for SLI in 7 of the 200 patients (3.5\%) with radiographic signs of SLI, as diagnosed by a radiologist. We found that of the 200 patients with a DRF and concern for 
Table 3 Characteristics of the patients who had treatment for their SL diastasis

\begin{tabular}{|c|c|c|c|c|}
\hline \multirow[t]{2}{*}{ Characteristics } & \multicolumn{4}{|c|}{ Patient with treatment for SL diastasis } \\
\hline & Patient 1 & Patient 2 & Patient 3 & Patient 4 \\
\hline Age in years & 52 & 56 & 64 & 53 \\
\hline Sex & q & $\sigma^{x}$ & $+q$ & $\sigma^{x}$ \\
\hline Reported obesity in medical chart & No & Yes & Yes & No \\
\hline Reported alcohol abuse in medical chart & No & No & No & No \\
\hline Reported tobacco abused in medical chart & No & No & No & No \\
\hline \multicolumn{5}{|l|}{ Trauma characteristics } \\
\hline \multicolumn{5}{|l|}{ Mechanism of Injury } \\
\hline Injured wrist & Dominant & Nondominant & Nondominant & Nondominant \\
\hline Open versus closed fracture & Closed & Closed & Closed & Closed \\
\hline \multicolumn{5}{|l|}{ Diagnosis and treatment characteristics } \\
\hline Time from injury to distal radius surgery & 16 days & 12 days & 5 days & 1 day \\
\hline $\begin{array}{l}\text { Time from injury to first radiograph } \\
\text { stating SL diastasis }\end{array}$ & 28 days & 0 days & 250 days $^{\mathrm{a}}$ & 0 days \\
\hline Type of DRFx treatment & ORIF & ORIF & ORIF & ORIF \\
\hline \multicolumn{5}{|l|}{ Radiographic characteristics } \\
\hline Type of radiograph for first diagnosis & Postreduction & Postreduction & Post-op follow-up & Prereduction \\
\hline Measured SL diastasis on first SL+ radiograph & $2.9 \mathrm{~mm}$ & $5.3 \mathrm{~mm}$ & $2.3 \mathrm{~mm}^{\mathrm{a}}$ & $4.3 \mathrm{~mm}$ \\
\hline
\end{tabular}

Abbreviations: DRF, distal radius fracture; ORIF, open reduction internal fixation; SL, scapholunate ligament.

${ }^{a}$ This patient had an intraoperatively discovered SL diastasis that was treated in the same procedure. The first radiograph where a radiologist mentioned a SL diastasis was 36 weeks post-op.

SLI by a radiologist, only 4 patients (2\%) received surgical repair of their SLI. Radiographical and clinical criteria for the diagnosis of SLI have both been extensively described. ${ }^{19}$ Despite these well-defined criteria, radiologists diagnosed SLI in 200 out of 2,923 patients who had ORIF of their DRF, whereas the surgeons treating these patients only identified four. Prior published data regarding SL surgery is mainly based on an arthroscopic diagnosis of SLI. ${ }^{1,4,5,14,20,21}$ Apart from institutional differences and a lack of consensus on the treatment of SLI, ${ }^{22}$ differences in rates of operative treatment of SLI may possibly be explained by different criteria for diagnosing SLI radiographically, ${ }^{23-26}$ differences in mean age between the study populations, and patient preferences.

It must also be considered that over the past 25 years, since the first reports of SLI in the setting of a DRF were published, our understanding of the pathology of this injury has evolved. Cadaveric studies have demonstrated that for SL diastasis to occur several ligamentous injuries have to occur at the same time, ${ }^{27}$ and that increased SL distance or altered carpal relationships do not necessarily indicate pathology. ${ }^{28}$ Since SLI in the setting of a DRF does not always lead to carpal malalignment, not every SLI may need treatment. Finally, as the treatment of DRFs has predominantly evolved to routine volar locked plating, one has to speculate that restoration of the anatomy of the radius is likely to have a positive influence on the carpus and its intercarpal relationships. More recent studies indicate that surgical repair of SLI is especially worthwhile in patients with (radiographic) signs of severe
SLI and/or a high functional demand of their injured wrist. $^{2,29}$

Overall, our findings indicate that the current abilities to radiographically diagnose SLI effectively in the setting of a DRF are suboptimal. Based on our data, it appears that radiographic diagnosis of SLI is challenging and often does not seem to correspond with clinical concerns by the treating surgeons. Radiologists demonstrate a low threshold to identify these injuries while the number of SLIs identified by the treating surgeon is a remarkably smaller number. The actual number of operative SL repair is only a fraction of the denominator of all DRFs.

As the understanding of this concurrence has evolved over the last three decades, treatment recommendations are also evolving. We no longer recommend routine exploration and repair for a radiographic appearance of SLI, noted on preoperative films. ${ }^{6,7}$ Rather, our current treatment practice consists of zero rotation posteroanterior and lateral radiographs of the contralateral wrist performed at the initial office visit (-Fig. 2). If there is a difference in SL distance, then the patient is alerted to the possibility of this injury. They are also cautioned about the need for its repair and that this may be associated with some degree of permanent loss of wrist motion. We then fix the DRF operatively. After fixation is complete, using fluoroscopy, the wrist is imaged during flexion and extension as well as during radial and ulnar deviation. If SL relationships are restored and the scaphoid and lunate move as one unit, no further treatment 


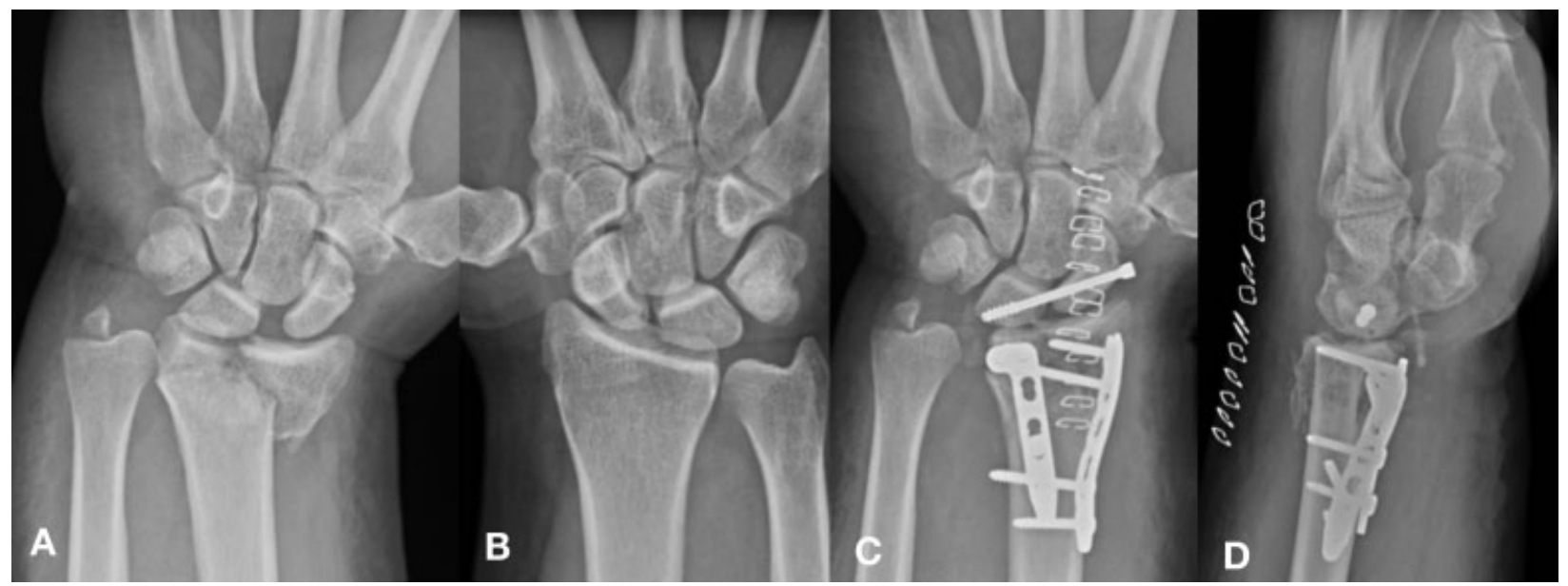

Fig. 2 A 56-year-old man presented with a left distal radius fracture (DRF) after a high-energy injury. (A) Preoperative radiograph shows a comminuted intra-articular fracture, with widening of the scapholunate ligament (SL) gap. (B) Radiograph of the uninjured wrist demonstrates normal SL relation. (C and D) Postoperative radiographs showing columnar plate fixation of the DRF. The SL relation was restored with a screw after SL ligament repair.

is recommended, but if there is a loss of the normal SL relationships in addition to an increased SL distance, then the SLI is operatively repaired.

\section{Authors' Contributions}

This study represents a great deal of effort, resources, and dedication on the part of the authors in reviewing and reconstructing all cases, reviewing the literature, and performing statistical analyses. All authors have participated in a material way to the following elements: Study design: S.Ö., J.K., F.B., C.M.; Gathered data: S.Ö., J.K., N.D.; Analyzed data: S.Ö., C.M., F.B., C.M.; Initial draft: S.Ö., N.D., F.B., C.M.; Ensured accuracy of data: S.Ö., J.K., N.D.

Note

This work was performed at the Hand and Upper Extremity Service, Department of Orthopedic Surgery, Massachusetts General Hospital, Harvard Medical School, Boston, Massachusetts.

\section{Ethical Approval}

The Institutional Review Board approved this study under protocol MGH/2009P001019.

\section{Funding}

This study received departmental funding from Hand and Upper Extermity Service, Massachusetts General Hospital, Harvard Medical School, Boston, MA.

\section{Conflict of Interest}

None.

\section{References}

1 Lindau $\mathrm{T}$, Arner $\mathrm{M}$, Hagberg L. Intraarticular lesions in distal fractures of the radius in young adults. A descriptive arthroscopic study in 50 patients. J Hand Surg [Br] 1997;22 (05):638-643
2 Jones VM, Everding NG, Desmarais JM, Soong MC. Scapholunate instability after distal radius volar plating. Hand (NY) 2015;10 (04):678-682

3 Finsen V, Rajabi B, Rod O, Roed K, Alm-Paulsen PS, Russwurm H. The clinical outcome after extra-articular colles fractures with simultaneous moderate scapholunate dissociation. J Wrist Surg 2014;3(02):123-127

4 Ogawa T, Tanaka T, Yanai T, Kumagai H, Ochiai N. Analysis of soft tissue injuries associated with distal radius fractures. BMC Sports Sci Med Rehabil 2013;5(01):19

5 Forward DP, Lindau TR, Melsom DS. Intercarpal ligament injuries associated with fractures of the distal part of the radius. J Bone Joint Surg Am 2007;89(11):2334-2340

6 Mudgal CS, Jones WA. Scapho-lunate diastasis: a component of fractures of the distal radius. J Hand Surg [Br] 1990;15(04): 503-505

7 Mudgal C, Hastings H. Scapho-lunate diastasis in fractures of the distal radius. Pathomechanics and treatment options. J Hand Surg [Br] 1993;18(06):725-729

8 Kitay A, Wolfe SW. Scapholunate instability: current concepts in diagnosis and management. J Hand Surg Am 2012;37(10): 2175-2196

9 Gradl G, Neuhaus V, Fuchsberger T, Guitton TG, Prommersberger KJ, Ring D; Science of Variation Group. Radiographic diagnosis of scapholunate dissociation among intra-articular fractures of the distal radius: interobserver reliability. J Hand Surg Am 2013;38 (09):1685-1690

10 Richards RS, Bennett JD, Roth JH, Milne K Jr. Arthroscopic diagnosis of intra-articular soft tissue injuries associated with distal radial fractures. J Hand Surg Am 1997;22(05):772-776

11 Wright TW, Del Charco M, Wheeler D. Incidence of ligament lesions and associated degenerative changes in the elderly wrist. J Hand Surg Am 1994;19(02):313-318

12 Chennagiri RJ, Lindau TR. Assessment of scapholunate instability and review of evidence for management in the absence of arthritis. J Hand Surg Eur Vol 2013;38(07):727-738

13 Mackenney PJ, McQueen MM, Elton R. Prediction of instability in distal radial fractures. J Bone Joint Surg Am 2006;88(09): 1944-1951

14 Geissler WB, Freeland AE, Savoie FH, McIntyre LW, Whipple TL. Intracarpal soft-tissue lesions associated with an intra-articular fracture of the distal end of the radius. J Bone Joint Surg Am 1996; 78(03):357-365 
15 Laulan J, Bismuth JP. Intracarpal ligamentous lesions associated with fractures of the distal radius: outcome at one year. A prospective study of 95 cases. Acta Orthop Belg 1999;65(04): 418-423

16 Tang JB, Shi D, Gu YQ, Zhang QG. Can cast immobilization successfully treat scapholunate dissociation associated with distal radius fractures? J Hand Surg Am 1996;21(04):583-590

17 Megerle K, Pöhlmann S, Kloeters O, Germann G, Sauerbier M. The significance of conventional radiographic parameters in the diagnosis of scapholunate ligament lesions. Eur Radiol 2011;21(01): 176-181

18 Pliefke J, Stengel D, Rademacher G, Mutze S, Ekkernkamp A, Eisenschenk A. Diagnostic accuracy of plain radiographs and cineradiography in diagnosing traumatic scapholunate dissociation. Skeletal Radiol 2008;37(02):139-145

19 Manuel J, Moran SL. The diagnosis and treatment of scapholunate instability. Hand Clin 2010;26(01):129-144

20 Kasapinova K, Kamiloski V. Influence of associated lesions of the intrinsic ligaments on distal radius fractures outcome. Arch Orthop Trauma Surg 2015;135(06):831-838

21 Peicha G, Seibert F, Fellinger M, Grechenig W. Midterm results of arthroscopic treatment of scapholunate ligament lesions associated with intra-articular distal radius fractures. Knee Surg Sports Traumatol Arthrosc 1999;7(05):327-333
22 Pappou IP, Basel J, Deal DN. Scapholunate ligament injuries: a review of current concepts. Hand (NY) 2013;8(02):146-156

23 Cautilli GP, Wehbé MA. Scapho-lunate distance and cortical ring sign. J Hand Surg Am 1991;16(03):501-503

24 Kindynis P, Resnick D, Kang HS, Haller J, Sartoris DJ. Demonstration of the scapholunate space with radiography. Radiology 1990 ; 175(01):278-280

25 Linscheid RL, Dobyns JH, Beabout JW, Bryan RS. Traumatic instability of the wrist. Diagnosis, classification, and pathomechanics. J Bone Joint Surg Am 1972;54(08):1612-1632

26 Moneim MS. The tangential posteroanterior radiograph to demonstrate scapholunate dissociation. J Bone Joint Surg Am 1981;63(08):1324-1326

27 Short WH, Werner FW, Green JK, Sutton LG, Brutus JP. Biomechanical evaluation of the ligamentous stabilizers of the scaphoid and lunate: part III. J Hand Surg Am 2007;32(03):297-309

28 Rimington TR, Edwards SG, Lynch TS, Pehlivanova MB. Intercarpal ligamentous laxity in cadaveric wrists. J Bone Joint Surg $\mathrm{Br} 2010$; 92(11):1600-1605

29 Gradl G, Pillukat T, Fuchsberger T, Knobe M, Ring D, Prommersberger KJ. The functional outcome of acute scapholunate ligament repair in patients with intraarticular distal radius fractures treated by internal fixation. Arch Orthop Trauma Surg 2013; 133(09):1281-1287 